\title{
Slow decay of dynamical correlation functions for nonequilibrium quantum states
}

\author{
Takayuki Miyadera \\ Department of Information Sciences \\ Tokyo University of Science \\ Noda City, Chiba 278-8510, Japan
}

\begin{abstract}
A property of dynamical correlation functions for nonequilibrium states is discussed. We consider arbitrary dimensional quantum spin systems with local interaction and translationally invariant states with nonvanishing current over them. A correlation function between local charge and local Hamiltonian at different spacetime points is shown to exhibit slow decay.
\end{abstract}

e-mail: miyadera@is.noda.tus.ac.jp 


\section{Introduction}

In this paper we treat nonequilibrium states of quantum spin systems. When we speak of nonequilibrium states, there are two kinds of states, that is, states near equilibrium and far from equilibrium. As for the investigation of the former nonequilibrium states, the main purpose is to understand how the states approach to an equilibrium state. The latter are states which, on the other hand, can not be treated as perturbed equilibrium states and do not converge to any equilibrium states, whose properties are less known. We, in this paper, study a property of the latter nonequilibrium states. There have been works on the nonequilibrium states from various points of view $[1,2,3,4,5,6,7,8,9,10]$. Among the various results, it is known that nonequilibrium steady states exhibit slow decay of equal-time correlation function from approximate theories like fluctuating hydrodynamics [1]. Recently an approach to the problem from exactly solvable models [2] has been investigated and the expected behavior of equal-time correlation function was confirmed.

In this paper, another type of slow decay of dynamical correlation function will be discussed. We consider locally interacting quantum spin systems and translationally invariant states with nonvanishing current over them. We do not impose any other conditions like stationarity of the states and do not ask how the nonequilibrium states are realized. Since the states have finite current at infinity, they cannot be locally perturbed equilibrium states and thus are far from equilibrium. We consider a correlation function between local charge and local Hamiltonian at different spacetime points. It will be shown in mathematically rigorous way that the correlation functions of the states exhibit slow

decay with respect to space and time. The way to show the claim is a generalization of the method employed in [10].

The paper is organized as follows. In section 2, we introduce our nonequilibrium states on quantum spin systems. In section 3 , the main theorem is proved.

\section{States with Nonvanishing Current on Quantum Spin Systems}

Let us consider a $d$-dimensional $(d=1,2,3, \cdots)$ infinite quantum spin system (see e.g., [11]). For simplicity, we assume nearest neighbor translationally invariant interaction. That is, for each pair of neighboring sites $\mathbf{x}$ and $\mathbf{y}$ (i.e., $\mathbf{x}, \mathbf{y} \in \mathbf{Z}^{d}$ s.t. $|\mathbf{x}-\mathbf{y}|=1$ ), a self adjoint operator $h(\mathbf{x}, \mathbf{y})$ is defined and satisfies $\tau_{\mathbf{z}}(h(\mathbf{x}, \mathbf{y}))=h(\mathbf{x}+\mathbf{z}, \mathbf{y}+\mathbf{z})$ for all $\mathbf{z} \in \mathbf{Z}^{d}$ where $\tau_{\mathbf{z}}$ stands for the translation operator. Denoting fundamental vector with direction $i$ as $\mathbf{e}_{i}$, local Hamiltonian with respect to a finite region $\Lambda \subset \mathbf{Z}$ is defined as

$$
H_{\Lambda}=\sum_{r=1}^{d} \sum_{\left\{\mathbf{x}, \mathbf{x}+\mathbf{e}_{r}\right\} \subset \Lambda} h\left(\mathbf{x}, \mathbf{x}+\mathbf{e}_{r}\right) .
$$


In this paper we employ the Heisenberg picture and the Hamiltonian defines time evolution of local operators, say $A$, by

$$
\alpha_{t}(A):=\lim _{\Lambda \rightarrow \mathbf{Z}^{d}} e^{i H_{\Lambda} t} A e^{-i H_{\Lambda} t}
$$

where the limit is taken with respect to norm topology (we put $\hbar=1$ ). To discuss (electric) current, a charge $n(\mathbf{x})$, a self-adjoint operator, is assumed to be defined on each site $\mathbf{x}$ [12], and the charge on a finite region $\Lambda$ is denoted as $N_{\Lambda}:=\sum_{\mathbf{x} \in \Lambda} n(\mathbf{x})$. It induces a symmetry transformation on each finite sublattice $\Lambda$, that is, $\left[H_{\Lambda}, N_{\Lambda}\right]=0$ holds. The current operator between sites $\mathbf{x}$ and $\mathbf{x}+\mathbf{e}_{r}$ is defined by $j\left(\mathbf{x}, \mathbf{x}+\mathbf{e}_{r}\right):=$ $i\left[n(\mathbf{x}), h\left(\mathbf{x}, \mathbf{x}+\mathbf{e}_{r}\right)\right]=-i\left[n\left(\mathbf{x}+\mathbf{e}_{r}\right), h\left(\mathbf{x}, \mathbf{x}+\mathbf{e}_{r}\right)\right]$.

The above seemingly abstract setting has physically interesting examples. For instance, interacting fermion system is on the list. For each $\mathbf{x} \in \mathbf{Z}^{d}$, charge is defined as $n(\mathbf{x}):=c_{\mathbf{x}}^{*} c_{\mathbf{x}}$ where $c_{\mathbf{x}}$ is a fermionic annihilation operator at site $\mathbf{x}$ and $h\left(\mathbf{x}, \mathbf{x}+\mathbf{e}_{r}\right)=-T\left(c_{\mathbf{x}+\mathbf{e}_{r}}^{*} c_{\mathbf{x}}+\right.$ $\left.c_{\mathbf{x}}^{*} c_{\mathbf{x}+\mathbf{e}_{r}}\right)+V n(\mathbf{x}) n\left(\mathbf{x}+\mathbf{e}_{r}\right)$ gives a nearest neighbor Hamiltonian where $T$ and $V$ are real constants. The current operator is calculated as $j\left(\mathbf{x}, \mathbf{x}+\mathbf{e}_{r}\right)=i T\left(c_{\mathbf{x}+\mathbf{e}_{r}}^{*} c_{\mathbf{x}}-c_{\mathbf{x}}^{*} c_{\mathbf{x}+\mathbf{e}_{r}}\right)$. The Heisenberg model can be another example. On each site $\mathbf{x} \in \mathbf{Z}$, spin operators $\left(S_{\mathbf{x}}^{(1)}, S_{\mathbf{x}}^{(2)}, S_{\mathbf{x}}^{(3)}\right)$ satisfying usual commutation relations for angular momentum live on.

$h\left(\mathbf{x}, \mathbf{x}+\mathbf{e}_{r}\right):=S_{\mathbf{x}}^{(1)} S_{\mathbf{x}+\mathbf{e}_{r}}^{(1)}+S_{\mathbf{x}}^{(2)} S_{\mathbf{x}+\mathbf{e}_{r}}^{(2)}+\lambda S_{\mathbf{x}}^{(3)} S_{\mathbf{x}+\mathbf{e}_{r}}^{(3)}(\lambda$ is a real constant $)$ and $n(\mathbf{x}):=S_{\mathbf{x}}^{(3)}$ leads the current $j\left(\mathbf{x}, \mathbf{x}+\mathbf{e}_{r}\right)=-S_{\mathbf{x}}^{(2)} S_{\mathbf{x}+\mathbf{e}_{r}}^{(1)}+S_{\mathbf{x}}^{(1)} S_{\mathbf{x}+\mathbf{e}_{r}}^{(2)}$.

In this paper, we consider translationally invariant states with nonvanishing current at time $t=0$. Without loss of generality, we can fix the first axis $\mathbf{e}_{1}$ as a direction of nonvanishing current. That is, we consider translationally invariant states which satisfy

$$
\left\langle j\left(\mathbf{0}, \mathbf{e}_{1}\right)\right\rangle \neq 0 .
$$

Since our conditions are weak, it surely contains a several physically interesting models $[3,4]$. However, we do not impose any other conditions like stationarity and stability, and therefore some states might be hardly realized in real physical situation [13]. The crucial point is that the states have nonvanishing current at infinity and they are not, say, locally perturbed equilibrium states.

\section{Slow decay of correlation function}

In derivation of slow decay of correlation functions for continuous symmetry breaking equilibrium states (Goldstone theorem), it is crucial to represent an order parameter by a commutator $[14,15]$. Our discussion can be considered as a nonequilibrium analogue of [14]. For nonequilibrium states, the following is an important observation. For finite sublattices $V$ and $\Lambda$ satisfying $V \supset \Lambda$,

$$
\left[-i \sum_{\mathbf{x} \in V} x_{1} n(\mathbf{x}), H_{\Lambda}\right]=\sum_{\left\{\mathbf{y}, \mathbf{y}+\mathbf{e}_{1}\right\} \subset \Lambda} j\left(\mathbf{y}, \mathbf{y}+\mathbf{e}_{1}\right)
$$

holds where $x_{1}$ is a component of $\mathbf{x}=\left(x_{1}, x_{2}, \cdots, x_{d}\right) \in \mathbf{Z}^{d}$. Hereafter, all the finite sublattices are assumed to be $d$-dimensional cubes, and we use simplified notations $H_{R}:=$ 
$H_{[-R, R]^{d}}$ for positive integer $R$ where $[-R, R]^{d}:=\left\{\mathbf{x}=\left(x_{1}, x_{2}, \cdots, x_{d}\right) \in \mathbf{Z}^{d} \mid-R \leq x_{j} \leq\right.$ $R$ for all $j=1,2, \cdots, d\}$. Taking expectation value of (1) with respect to a translationally invariant state and we put $\Lambda=[-R, R]^{d}$, take $W>R$ and divide (1) by $\mid\left\{\mathbf{y} \mid\left\{\mathbf{y}, \mathbf{y}+\mathbf{e}_{1}\right\} \subset\right.$ $\left.[-R, R]^{d}\right\} \mid=2 R(2 R+1)^{d-1}$. Then we obtain

$$
\frac{1}{2 R(2 R+1)^{d-1}}\left\langle\left[-i \sum_{\mathbf{x} \in[-W, W]^{d}} x_{1} n(\mathbf{x}), H_{R}\right]\right\rangle=\left\langle j\left(\mathbf{0}, \mathbf{e}_{1}\right)\right\rangle
$$

where we have used the translational invariance of the state. By letting the size of cubic lattices in the above equation infinity, we obtain

$$
\lim _{R \rightarrow \infty} \lim _{W \rightarrow \infty} \frac{1}{2 R(2 R+1)^{d-1}}\left\langle\left[-i \sum_{\mathbf{x} \in[-W, W]^{d}} x_{1} n(\mathbf{x}), H_{R}\right]\right\rangle=\left\langle j\left(\mathbf{0}, \mathbf{e}_{1}\right)\right\rangle .
$$

The ordering of the above limiting procedures is crucial and can not be exchanged. In fact it is easy to see that if we take $R \rightarrow \infty$ first, for steady states it always gives zero. Moreover we can show the following lemma.

Lemma 1 For an arbitrary translationally invariant state and for any time $t \in \mathbf{R}$,

$$
\lim _{R \rightarrow \infty} \lim _{W \rightarrow \infty} \frac{1}{2 R(2 R+1)^{d-1}}\left\langle\left[-i \sum_{\mathbf{x} \in[-W . W]^{d}} x_{1} n(\mathbf{x}), \alpha_{t}\left(H_{R}\right)\right]\right\rangle=\left\langle j\left(\mathbf{0}, \mathbf{e}_{1}\right)\right\rangle
$$

holds, where $\alpha_{t}\left(H_{R}\right)$ is a time evolved object of $H_{R}$ (remind that we are in the Heisenberg picture). Although the lhs includes time $t$ explicitly, its value does not depend on $t$.

\section{Proof:}

Since for $t=0$, the above equation holds (see (2)), we estimate its deviation for finite $t$. That is, since

$$
\begin{aligned}
& \frac{1}{2 R(2 R+1)^{d-1}}\left[-i \sum_{\mathbf{x} \in[-W, W]^{d}} x_{1} n(\mathbf{x}), \alpha_{t}\left(H_{R}\right)\right] \\
= & \frac{1}{2 R(2 R+1)^{d-1}}\left[-i \sum_{\mathbf{x} \in[-W, W]^{d}} x_{1} n(\mathbf{x}), H_{R}\right] \\
+ & \frac{1}{2 R(2 R+1)^{d-1}} \int_{0}^{t} d s\left[-i \sum_{\mathbf{x} \in[-W, W]^{d}} x_{1} n(\mathbf{x}),-i \alpha_{s}\left(\left[H_{R}, H_{R+1}\right]\right)\right]
\end{aligned}
$$

holds, to show

$$
\lim _{R \rightarrow \infty} \lim _{W \rightarrow \infty} \frac{1}{2 R(2 R+1)^{d-1}}\left\|\left[-i \sum_{\mathbf{x} \in[-W, W]^{d}} x_{1} n(\mathbf{x}),-i \alpha_{s}\left(\left[H_{R}, H_{R+1}\right]\right)\right]\right\|=0
$$


proves our lemma. Let us consider first the case $s=0$ in the above equation.

$$
\begin{aligned}
& \frac{1}{2 R(2 R+1)^{d-1}}\left[-i \sum_{\mathbf{x} \in[-W, W]^{d}} x_{1} n(\mathbf{x}),-i\left[H_{R}, H_{R+1}\right]\right] \\
= & \frac{1}{2 R(2 R+1)^{d-1}}\left[H_{R},\left[H_{R+1}, \sum_{\mathbf{x} \in[-W, W]^{d}} x_{1} n(\mathbf{x})\right]\right] \\
+ & \frac{1}{2 R(2 R+1)^{d-1}}\left[H_{R+1},\left[\sum_{\mathbf{x} \in[-W, W]^{d}} x_{1} n(\mathbf{x}), H_{R}\right]\right]
\end{aligned}
$$

which is obtained by Jacobi identity can be rewritten as, by letting $W \rightarrow \infty$,

$$
\begin{aligned}
\lim _{W \rightarrow \infty}(4)= & \frac{1}{2 R(2 R+1)^{d-1}}\left\{\left[H_{R+1},-i \sum_{\left\{\mathbf{x}, \mathbf{x}+\mathbf{e}_{1}\right\} \subset[-R-1, R+1]^{d} \backslash[-R, R]^{d}} j\left(\mathbf{x}, \mathbf{x}+\mathbf{e}_{1}\right)\right]\right. \\
& \left.-\left[H_{R+1}-H_{R},-i \sum_{\left\{\mathbf{x}, \mathbf{x}+\mathbf{e}_{1}\right\} \subset[-R-1, R+1]^{d}} j\left(\mathbf{x}, \mathbf{x}+\mathbf{e}_{1}\right)\right]\right\},
\end{aligned}
$$

whose norm is estimated as $O(1 / R)$ and goes to zero as $R \rightarrow \infty$. Next we consider the case of finite $s$ in (3). Thanks to

$$
\begin{aligned}
& \frac{1}{2 R(2 R+1)^{d-1}}\left\|\left[-i \sum_{\mathbf{x} \in[-W, W]^{d}} x_{1} n(\mathbf{x}),-i \alpha_{s}\left(\left[H_{R}, H_{R+1}\right]\right)\right]\right\| \\
= & \frac{1}{2 R(2 R+1)^{d-1}}\left\|\left[-i \sum_{\mathbf{x} \in[-W, W]^{d}} x_{1} \alpha_{-s}(n(\mathbf{x})),-i\left[H_{R}, H_{R+1}\right]\right]\right\| \\
\leq & \frac{1}{2 R(2 R+1)^{d-1}}\left\|\left[-i \sum_{\mathbf{x} \in[-W, W]^{d}} x_{1} n(\mathbf{x}),-i\left[H_{R}, H_{R+1}\right]\right]\right\| \\
+ & \frac{1}{2 R(2 R+1)^{d-1}}\left|\int_{-s}^{0} d u\left\|\left[-i \sum_{\mathbf{x} \in[-W, W]^{d}} x_{1} \frac{d \alpha_{u}(n(\mathbf{x}))}{d u},-i\left[H_{R}, H_{R+1}\right]\right]\right\|\right|
\end{aligned}
$$

to show that the term

$$
\frac{1}{2 R(2 R+1)^{d-1}}\left\|\left[-i \sum_{\mathbf{x} \in[-W, W]^{d}} x_{1} \frac{d \alpha_{u}(n(\mathbf{x}))}{d u},-i\left[H_{R}, H_{R+1}\right]\right]\right\|
$$

for each $u$ converges to zero as $W \rightarrow \infty$ and $R \rightarrow \infty$ proves the claim. Since $\frac{d \alpha_{u}(n(\mathbf{x}))}{d u}=$ $\sum_{l=1}^{d}\left(\alpha_{u}\left(j\left(\mathbf{x}-\mathbf{e}_{l}, \mathbf{x}\right)\right)-\alpha_{u}\left(j\left(\mathbf{x}, \mathbf{x}+\mathbf{e}_{l}\right)\right)\right)$ holds, it follows that

$$
\begin{aligned}
& \sum_{\mathbf{x} \in[-W, W]^{d}} x_{1} \frac{d \alpha_{u}(n(\mathbf{x}))}{d u} \\
= & \sum_{-W \leq x_{2}, \cdots, x_{d} \leq W}\left\{-W \alpha_{u}\left(j\left(-W-1, x_{2}, \cdots, x_{d},-W, x_{2}, \cdots, x_{d}\right)\right)\right.
\end{aligned}
$$




$$
\begin{aligned}
& \left.-W \alpha_{u}\left(j\left(W, x_{2}, \cdots, x_{d}, W+1, x_{2}, \cdots, x_{d}\right)\right)+\sum_{x_{1}=-W}^{W-1} \alpha_{u}\left(j\left(\mathbf{x}, \mathbf{x}+\mathbf{e}_{1}\right)\right)\right\} \\
& +\sum_{l=2-W \leq x_{1}, x_{2}, \cdots, x_{l-1}, x_{l+1}, \cdots, x_{d} \leq W}^{d} \\
& \quad x_{1}\left\{\alpha_{u}\left(j\left(x_{1}, x_{2}, \cdots, x_{l-1},-W-1, x_{l+1}, \cdots, x_{d}, x_{1}, \cdots,-W, \cdots, x_{d}\right)\right)\right. \\
& \left.-\quad \alpha_{u}\left(j\left(x_{1}, x_{2}, \cdots, x_{l-1}, W, x_{l+1}, \cdots, x_{d}, x_{1}, \cdots, W+1, \cdots, x_{d}\right)\right)\right\}
\end{aligned}
$$

holds. We employ repeatedly the following group velocity lemma (Theorem 6.2.11 of [11]). There exists a positive constant $V$ such that for strictly local observables $A$ and $B$ the following inequality holds:

$$
\left\|\left[A, \alpha_{t} \circ \tau_{\mathbf{x}}(B)\right]\right\| \leq C\|A\|\|B\| \exp (-|x|+V|t|),
$$

where $C$ is a constant depending only on size of the regions where $A$ and $B$ live in. Let us substitute (6) for (5) and estimate its each term. Thanks to $\left\|-i\left[H_{R}, H_{R+1}\right]\right\|=O\left(R^{d-1}\right)$,

$$
\begin{aligned}
& \frac{1}{2 R(2 R+1)^{d-1}} \|\left[\sum_{-W \leq x_{2}, \cdots, x_{d} \leq W}\right. \\
& \left.-W \alpha_{u}\left(j\left(-W-1, x_{2}, \cdots, x_{d},-W, x_{2}, \cdots, x_{d}\right)\right),-i\left[H_{R}, H_{R+1}\right]\right] \| \\
\leq & O\left(\frac{1}{R}\right) O\left(W^{d}\right) \exp (-(W-R)+V|u|)
\end{aligned}
$$

holds and it goes to zero as $W \rightarrow \infty$. The commutator related with $-W \alpha_{u}\left(j\left(W, x_{2}, \cdots, x_{d}, W+1, x_{2}, \cdots, x_{d}\right)\right)$ also vanishes in the same manner. Next one can see that

$$
\begin{aligned}
& \frac{1}{2 R(2 R+1)^{d-1}}\left\|\left[\sum_{x_{1}=-W}^{W-1} \sum_{-W \leq x_{2}, \cdots, x_{d} \leq W} \alpha_{u}\left(j\left(\mathbf{x}, \mathbf{x}+\mathbf{e}_{1}\right)\right),-i\left[H_{R}, H_{R+1}\right]\right]\right\| \\
\leq & \left(\sum_{\mathbf{x} \in \mathbf{Z}^{d}} e^{-|\mathbf{x}|}\right) e^{V|u|} O\left(\frac{1}{R}\right)
\end{aligned}
$$

holds and it goes to zero as $R \rightarrow \infty$. Finally,

$$
\begin{aligned}
& \frac{1}{2 R(2 R+1)^{d-1} \|\left[\sum_{-W \leq x_{1}, x_{2}, \cdots, x_{l-1}, x_{l+1}, \cdots, x_{d} \leq W}\right.} \\
& x_{1} \alpha_{u}\left(j\left(x_{1}, x_{2}, \cdots, x_{l-1},-W-1, x_{l+1}, \cdots, x_{d}, x_{1}, \cdots,-W, \cdots, x_{d}\right)\right), \\
& \left.-i\left[H_{R}, H_{R+1}\right]\right] \| \\
\leq & O\left(\frac{1}{R}\right) O\left(W^{d}\right) e^{-(W-R)+V|u|}
\end{aligned}
$$

holds and it goes to zero as $W \rightarrow \infty$ and the term related with $x_{l}=W$ vanishes similarly. Thus we proved the equation,

$$
\lim _{R \rightarrow \infty} \lim _{W \rightarrow \infty} \frac{1}{2 R(2 R+1)^{d-1}}\left\langle\left[-i \sum_{\mathbf{x} \in[-W, W]^{d}} x_{1} n(\mathbf{x}), H_{R}(t)\right]\right\rangle=\left\langle j\left(\mathbf{0}, \mathbf{e}_{1}\right)\right\rangle .
$$


Q.E.D.

By using an expression $H_{R}=\sum_{r=1}^{d} \sum_{-R \leq y_{r} \leq R-1} \sum_{-R \leq y_{1}, \cdots, y_{r-1}, y_{r+1}, \cdots, y_{d} \leq R} h\left(\mathbf{y}, \mathbf{y}+\mathbf{e}_{r}\right)$, one obtains for translationally invariant states,

$$
\begin{aligned}
\left\langle j\left(\mathbf{0}, \mathbf{e}_{1}\right)\right\rangle= & \lim _{R \rightarrow \infty} \frac{-i}{2 R(2 R+1)^{d-1}} \sum_{\mathbf{x} \in \mathbf{Z}^{d}} \sum_{r=1}^{d} \sum_{-R \leq y_{r} \leq R-1} \sum_{-R \leq y_{1}, \cdots, y_{r-1}, y_{r+1}, \cdots, y_{d} \leq R} \\
& x_{1}\left\langle\left[n(\mathbf{x}), \alpha_{t}\left(h\left(\mathbf{y}, \mathbf{y}+\mathbf{e}_{r}\right)\right)\right]\right\rangle
\end{aligned}
$$

from lemma 1. Thanks to the translational invariance of the state, substitution $\mathbf{z}:=\mathbf{y}-\mathbf{x}$ makes the above equation

$$
\begin{aligned}
\left\langle j\left(\mathbf{0}, \mathbf{e}_{1}\right)\right\rangle= & \lim _{R \rightarrow \infty} \frac{-i}{2 R(2 R+1)^{d-1}} \sum_{\mathbf{z} \in \mathbf{Z}^{d}} \sum_{r=1}^{d} \sum_{-R \leq y_{r} \leq R-1} \sum_{-R \leq y_{1}, \cdots, y_{r-1}, y_{r+1}, \cdots, y_{d} \leq R} \\
& \left(y_{1}-z_{1}\right)\left\langle\left[n(\mathbf{0}), \alpha_{t}\left(h\left(\mathbf{z}, \mathbf{z}+\mathbf{e}_{r}\right)\right)\right]\right\rangle .
\end{aligned}
$$

One can perform summation for $\mathbf{y}$ of the above equation. For $r=1$, one obtains

$$
\frac{-i}{2 R(2 R+1)^{d-1}} \sum_{-R \leq y_{1} \leq R-1} \sum_{-R \leq y_{2}, \cdots, y_{d} \leq R}\left(y_{1}-z_{1}\right)=i\left(z_{1}+\frac{1}{2}\right),
$$

and for $r \neq 1$,

$$
\frac{-i}{2 R(2 R+1)^{d-1}} \sum_{-R \leq y_{r} \leq R-1} \sum_{-R \leq y_{1}, \cdots, y_{r-1}, y_{r+1}, \cdots, y_{d} \leq R}\left(y_{1}-z_{1}\right)=i z_{1} .
$$

Thus we obtain

$$
\begin{aligned}
\left\langle j\left(\mathbf{0}, \mathbf{e}_{1}\right)\right\rangle= & i \sum_{\mathbf{z} \in \mathbf{Z}^{d}}\left\{\left(z_{1}+\frac{1}{2}\right)\left\langle\left[n(\mathbf{0}), \alpha_{t}\left(h\left(\mathbf{z}, \mathbf{z}+\mathbf{e}_{1}\right)\right)\right]\right\rangle\right. \\
& \left.+i \sum_{r=2}^{d} z_{1}\left\langle\left[n(\mathbf{0}), \alpha_{t}\left(h\left(\mathbf{z}, \mathbf{z}+\mathbf{e}_{r}\right)\right)\right]\right\rangle\right\} .
\end{aligned}
$$

Thanks to this representation, we obtain the following theorem.

Theorem 1 For translationally invariant states with nonvanishing current, there exists at least one $r \in\{1,2, \cdots, d\}$ such that neither $\left\langle\left[n(\mathbf{0}), \alpha_{t}\left(h\left(\mathbf{z}, \mathbf{z}+\mathbf{e}_{r}\right)\right)\right]\right\rangle$ nor a dynamical correlation function

$$
\left\langle n(\mathbf{0}) \alpha_{t}\left(h\left(\mathbf{z}, \mathbf{z}+\mathbf{e}_{r}\right)\right)\right\rangle^{T}:=\left\langle n(\mathbf{0}) \alpha_{t}\left(h\left(\mathbf{z}, \mathbf{z}+\mathbf{e}_{r}\right)\right)\right\rangle-\langle n(\mathbf{0})\rangle\left\langle\alpha_{t}\left(h\left(\mathbf{z}, \mathbf{z}+\mathbf{e}_{r}\right)\right)\right\rangle
$$

are absolutely integrable with respect to $\mathbf{z}$ and $t$.

Proof: First let us note that if $\left\langle\left[n(\mathbf{0}), \alpha_{t}\left(h\left(\mathbf{z}, \mathbf{z}+\mathbf{e}_{r}\right)\right)\right]\right\rangle$ is not absolutely integrable, $\left\langle n(\mathbf{0}) \alpha_{t}\left(h\left(\mathbf{z}, \mathbf{z}+\mathbf{e}_{r}\right)\right)\right\rangle^{T}$ is not also absolutely integrable. In fact thanks to

$$
\left\langle n(\mathbf{0}) \alpha_{t}\left(h\left(\mathbf{z}, \mathbf{z}+\mathbf{e}_{r}\right)\right)\right\rangle^{T}-\overline{\left\langle n(\mathbf{0}) \alpha_{t}\left(h\left(\mathbf{z}, \mathbf{z}+\mathbf{e}_{r}\right)\right)\right\rangle^{T}}=\left\langle\left[n(\mathbf{0}), \alpha_{t}\left(h\left(\mathbf{z}, \mathbf{z}+\mathbf{e}_{r}\right)\right)\right]\right\rangle,
$$


$\left|\left\langle\left[n(\mathbf{0}), \alpha_{t}\left(h\left(\mathbf{z}, \mathbf{z}+\mathbf{e}_{r}\right)\right)\right]\right\rangle\right| \leq 2\left|\left\langle n(\mathbf{0}) \alpha_{t}\left(h\left(\mathbf{z}, \mathbf{z}+\mathbf{e}_{r}\right)\right)\right\rangle^{T}\right|$ is obtained. Now we show that there exists $r$ such that $\left\langle\left[n(\mathbf{0}), \alpha_{t}\left(h\left(\mathbf{z}, \mathbf{z}+\mathbf{e}_{r}\right)\right)\right]\right\rangle$ is not absolutely integrable. From (7), one can see that

$$
\begin{aligned}
\left|\left\langle j\left(\mathbf{0}, \mathbf{e}_{1}\right)\right\rangle\right| \leq & \sum_{\mathbf{z} \in \mathbf{Z}^{d}}\left\{\left|z_{1}+\frac{1}{2}\right|\left|\left\langle\left[n(\mathbf{0}), \alpha_{t}\left(h\left(\mathbf{z}, \mathbf{z}+\mathbf{e}_{1}\right)\right)\right]\right\rangle\right|\right. \\
& \left.+\sum_{r=2}^{d}\left|z_{1}\right|\left|\left\langle\left[n(\mathbf{0}), \alpha_{t}\left(h\left(\mathbf{z}, \mathbf{z}+\mathbf{e}_{r}\right)\right)\right]\right\rangle\right|\right\} \\
\leq & \sum_{\mathbf{z} \in \mathbf{Z}^{d}}\left(\left|z_{1}\right|+\frac{1}{2}\right) \sum_{r=1}^{d}\left|\left\langle\left[n(\mathbf{0}), \alpha_{t}\left(h\left(\mathbf{z}, \mathbf{z}+\mathbf{e}_{r}\right)\right)\right]\right\rangle\right|
\end{aligned}
$$

holds. Thanks to the group velocity lemma, there exists a constant $C$ and $V$ such that

$$
\left|\left\langle\left[n(\mathbf{0}), \alpha_{t}\left(h\left(\mathbf{z}, \mathbf{z}+\mathbf{e}_{r}\right)\right)\right]\right\rangle\right| \leq C\|n(\mathbf{0})\|\left\|h\left(\mathbf{0}, \mathbf{0}+\mathbf{e}_{r}\right)\right\| \exp (-|\mathbf{z}|+V|t|)
$$

holds. Therefore, for an arbitrary $\epsilon>0$, there exists $N>0$ satisfying

$$
\sum_{|\mathbf{z}|>V|t|+N}\left(\left|z_{1}\right|+\frac{1}{2}\right) \sum_{r=1}^{d}\left|\left\langle\left[n(\mathbf{0}), \alpha_{t}\left(h\left(\mathbf{z}, \mathbf{z}+\mathbf{e}_{r}\right)\right)\right]\right\rangle\right|<\epsilon .
$$

Thus we obtain

$$
\begin{aligned}
\left|\left\langle j\left(\mathbf{0}, \mathbf{e}_{1}\right)\right\rangle\right| & \leq \sum_{\mathbf{z} \in \mathbf{Z}^{d}}\left(\left|z_{1}\right|+\frac{1}{2}\right) \sum_{r=1}^{d}\left|\left\langle\left[n(\mathbf{0}), \alpha_{t}\left(h\left(\mathbf{z}, \mathbf{z}+\mathbf{e}_{r}\right)\right)\right]\right\rangle\right| \\
& \leq \epsilon+\left\{V|t|+N+\frac{1}{2}\right\} \sum_{r=1}^{d} \sum_{|\mathbf{z}| \leq V|t|+N}\left|\left\langle\left[n(\mathbf{0}), \alpha_{t}\left(h\left(\mathbf{z}, \mathbf{z}+\mathbf{e}_{r}\right)\right)\right]\right\rangle\right| \\
& \leq \epsilon+\left(V|t|+N+\frac{1}{2}\right) \sum_{r=1}^{d} \sum_{\mathbf{z} \in \mathbf{Z}^{d}}\left|\left\langle\left[n(\mathbf{0}), \alpha_{t}\left(h\left(\mathbf{z}, \mathbf{z}+\mathbf{e}_{r}\right)\right)\right]\right\rangle\right|,
\end{aligned}
$$

which leads to

$$
\sum_{r=1}^{d} \sum_{\mathbf{z} \in \mathbf{Z}^{d}}\left|\left\langle\left[n(\mathbf{0}), \alpha_{t}\left(h\left(\mathbf{z}, \mathbf{z}+\mathbf{e}_{r}\right)\right)\right]\right\rangle\right| \geq \frac{\left|\left\langle j\left(\mathbf{0}, \mathbf{e}_{1}\right)\right\rangle\right|-\epsilon}{V|t|+N+1 / 2} .
$$

If for all $r=1,2, \cdots, d,\left|\left\langle\left[n(\mathbf{0}), \alpha_{t}\left(h\left(\mathbf{z}, \mathbf{z}+\mathbf{e}_{r}\right)\right)\right]\right\rangle\right|$ are integrable, their summation $\sum_{r=1}^{d}\left|\left\langle\left[n(\mathbf{0}), \alpha_{t}\left(h\left(\mathbf{z}, \mathbf{z}+\mathbf{e}_{r}\right)\right)\right]\right\rangle\right|$ must be integrable. It ends the proof. $\quad$ Q.E.D.

\section{Conclusion and Outlook}

In this paper, we considered states over $d$-dimensional infinite spin systems which are translationally invariant and have non-vanishing current expectations. The dynamical correlation function between $n(\mathbf{0})$ and $\alpha_{t}\left(h\left(\mathbf{x}, \mathbf{x}+\mathbf{e}_{r}\right)\right)$ for some $r$ shows slow decay with 
respect to space and time $(\mathbf{x}, t)$. The key observation to prove our theorem is to express the current operator by a commutator and to apply the argument of [14] for the continuous symmetry breaking case to our nonequilibrium case, although no continuous symmetry breaking takes place in our case. The behavior of dynamical correlation function is related with observable effect through response functions, which will be discussed elsewhere. Since the conditions for our nonequilibrium states are weak, our result is general but is not very strong. It is interesting to investigate whether additional physical conditions can derive more detailed form of the correlation function.

\section{Acknowledgment}

The author would like to thank anonymous referees for fruitful comments.

\section{References}

[1] J. R. Dorfman, T. R. Kirkpatrick, and J. V. Sengers, Annu. Rev Phys. Chem. 45 213 (1994).

[2] B. Derrida, J.L. Lebowitz, and E. R. Speer, Phys. Rev. Lett. 87150601 (2001).

[3] T. G. Ho and H. Araki, Tr. Mat. Inst. Steklova 228191 (2000).

[4] S. Tasaki, Chaos Solitons and Fractals 12,2657 (2001).

[5] A. Shimizu, J. Phys. Soc. Jpn. 65,1162 (1996).

[6] D. Buchholz, I. Ojima, and H. Roos, Ann. Physics 297,219 (2002).

[7] A. Yu. Alekseev, V. V. Cheianov, and J. Fröhlich, Phys. Rev. Lett. 81, 3503 (1998).

[8] V. Jaksic and C.-A. Pillet, J. Stat. Phys. 108,787 (2002).

[9] S. Sasa and H. Tasaki, Steady state thermodynamics for heat conduction, condmat/0108365

[10] T. Miyadera, Interdisciplinary Information Sciences 10 (1) 23 (2004)

[11] O. Bratteli and D. W. Robinson, Operator algebras and quantum statistical mechanics. 2. Equilibrium states. Models in quantum statistical mechanics. Texts and Monographs in Physics. Springer-Verlag, Berlin (1981).

[12] In the absence of charge operator, energy current can be defined from local Hamiltonian. The discussion in this paper holds also for the case when states have nonvanishing energy current. 
[13] In real experimental circumstance, nonequilibrium states are often realized by a several reservoirs attached to boundary of a finite system. The systems considered in this paper do not have boundary and might be regarded as a "thermodynamic limit" of such a real system. We do not know conditions which justify this point of view.

[14] M. Requardt, J. Math. Phys. 43, 351. (2002); J. Phys. A: Math. Gen. 131769 (1980).

[15] L. Landau, J. Fernando Perez and W. Wreszinski, J.Stat.Phys.26,755(1981) 